Check for updates

Cite this: RSC Adv., 2018, 8, 23241

Received 16th May 2018

Accepted 20th June 2018

DOI: $10.1039 / c 8 r a 04195 d$

rsc.li/rsc-advances

\section{Microwave assisted one-pot green synthesis of cinnoline derivatives inside natural sporopollenin microcapsules $\dagger$}

\author{
Amro K. F. Dyab (D) * and Kamal Usef Sadek (D) *
}

We present a green and efficient approach for the synthesis of novel cinnoline derivatives inside natural Lycopodium clavatum sporopollenin (LCS) microcapsules via a one-pot microwave (MW) assisted reaction for the first time. We also propose the concept that the robust micrometre-sized sporopollenin microcapsules can act as MW microreactors. We demonstrate the feasibility of this concept by in situ synthesising 8-hydroxy-7-nitro-6-(3-nitrophenyl)-3-oxo-2-(p-tolyl)-2,3,5,6-tetrahydrocinnoline-4carbonitrile inside the LCS microcapsules via a microwave (MW) assisted reaction of ethyl 5-cyano-4methyl-6-oxo-1-(p-tolyl)-1,6-dihydropyridazine-3-carboxylate with 1-nitro-2-phenylethylene in the presence of piperidine as a base at $100{ }^{\circ} \mathrm{C}$ for 20 minutes. The LCS microparticles are extensively characterised before and after the MW induced reaction using several techniques. The formation of the cinnoline compound inside the LCS microcapsules is confirmed by laser scanning confocal microscopy (LSCM), X-ray diffraction (XRD) and fourier-transform infrared spectroscopy (FTIR) analyses. Using liquid chromatography-mass spectrometry (LCMS) analyses, we show that the structural integrity of the cinnoline compound, recovered from the cinnoline loaded (cinn-loaded) LCS, is preserved. The pure cinnoline is found to show promising optical properties with two $\lambda_{\max }$ absorption peaks at 310 and $610 \mathrm{~nm}$. Both the pure cinnoline and cinn-loaded LCS show promising antibacterial activity against Pseudomonas aeruginosa (Gram-negative) and Bacillus cereus (Gram-negative) human pathogenic bacterial strains. The successful MW induced reaction of the prominent cinnoline derivative inside the biocompatible LCS microreactors can open up intriguing applications in materials and pharmaceutical sciences.

\section{Introduction}

Nature produces large quantities of fabulously complex and highly reliable pollen grains that protect the reproductive genetic constituents of male flowers from severe environmental conditions such as dehydration, UV light, microbial attack and high temperatures. ${ }^{1}$ The outer layer of the pollen represents the sporopollenin biopolymer, which has been described as the "diamond of the plant world"., ${ }^{2,3}$ The nitrogen-free sporopollenin can preserve the ornamental integrity after the extraction of the cytoplasmic core from the raw spores by simple acidic and alkaline treatments. ${ }^{4-6}$ In addition, the core removal produces allergen-free sporopollenin having a large cage ready for encapsulation. ${ }^{7}$ Due to its remarkable resilience, biocompatibility, uniformity in size and abundancy, sporopollenin has recently gained intriguing attention in different potential applications acting as either microparticles (as a solid support) ${ }^{\mathbf{8 - 1 4}}$ or

Chemistry Department, Faculty of Science, Minia University, Minia 61519, Egypt. E-mail: amro.dyab@mu.edu.eg; kusadek@yahoo.com

$\dagger$ Electronic supplementary information (ESI) available. See DOI: 10.1039/c8ra04195d microcapsules (for various encapsulations of materials)., ${ }^{2,715-18}$ The majority of research involving sporopollenin has utilised the thick-shelled $(\sim 1.5 \mu \mathrm{m})$ S-type species of Lycopodium clavatum L. spores (common club moss). Moreover, L. clavatum sporopollenin (LCS) have recently shown promising capabilities in encapsulation of drugs, ${ }^{7,19}$ live cells, ${ }^{18}$ oils, ${ }^{16,20}$ proteins $^{17}$ and inorganic nanoparticles. ${ }^{15}$ Materials can be encapsulated inside the cavity of sporopollenin via passive or vacuum diffusion through the possible nanochannels on their surfaces. ${ }^{17,21}$ Another possible route is by compressing sporopollenin into tablets, particularly for those species with scars on their surfaces. ${ }^{15,18,22}$ Over the past two decades, the microwave (MW) heating technique, described as "the Bunsen burner of the $21^{\text {st }}$ century", ${ }^{23}$ has proven to be an invaluable alternative in diverse applications such as, inter alia, organic and peptide synthesis, materials science, polymers, nanotechnology and biochemical methods. ${ }^{24}$ The advantages of MW over the conventional techniques was attributed to the fact that the former significantly reduces the reaction time, enhances product purities and provides high yields and selectivity. ${ }^{23,24}$ Unlike the aforementioned studies, we demonstrate here, for the first time, a significantly streamlined eco-friendly route for the in situ synthesis of novel cinnoline 
derivatives inside sporopollenin cages via a chemical reaction triggered by microwave irradiation. Our approach might also bridge the revolutionised microwave assisted organic synthesis and the versatile microencapsulation capability of natural biomaterials in an effort to open up various new potential applications. Most of heterocycles exhibit biological activities and are therefore of great importance for organic synthesis. ${ }^{25}$ Cinnoline derivatives are an important class of N-containing heteroaromatics and found intriguing interest as a privileged structures utilised as potential anticancer, ${ }^{26,27}$ anti-inflammatory ${ }^{28}$ antibacterial, ${ }^{29}$ fluorescent agents ${ }^{30}$ cell imaging ${ }^{31}$ and in designing liquid crystals. ${ }^{32,33}$ We have used in the current study a novel polyfunctional cinnoline derivative based on the reaction of ethyl 5-cyano-4-methyl-1-aryl-6-oxo-1,6-dihydropyridazine-3carboxylate with nitroolefins (Scheme 1) under controlled microwave irradiation that has been recently introduced by Sadek et al. $^{34}$ These novel cinnoline derivatives are inevitably entail more exploration of their promising physico-chemical properties and potential applications. Major questions are that whether the internal surface of the LCS has any role in a chemical reaction performed within their cores and the feasibility of these microcapsules to encapsulate and eventually recover intact materials. As shown in Scheme 1, our protocol involves the following steps: (i) extraction of empty LCS microcapsules from the raw spores; (ii) loading the dissolved reactants into the LCS by passive diffusion; (iii) promoting the reaction by MW irradiation at $100{ }^{\circ} \mathrm{C}$ for $20 \mathrm{~min}$; (iv) filtration of the product-loaded LCS microcapsules, washing with pure solvent to remove excess reagents and drying. Physico-chemical characterisations of the LCS before and after the loading process were investigated using several techniques to verify the successful formation of the reaction product inside the LCS microcapsules. We have also investigated the optical and thermal properties of the pure cinnoline derivative. In addition, the potential antibacterial activity of both pure cinnoline and the cinnoline-loaded LCS were explored. In light of the prominence of both cinnolines and natural biopolymers, our preliminary promising findings based on the above expedient green technique can open up several interesting potential applications in material and pharmaceutical sciences.

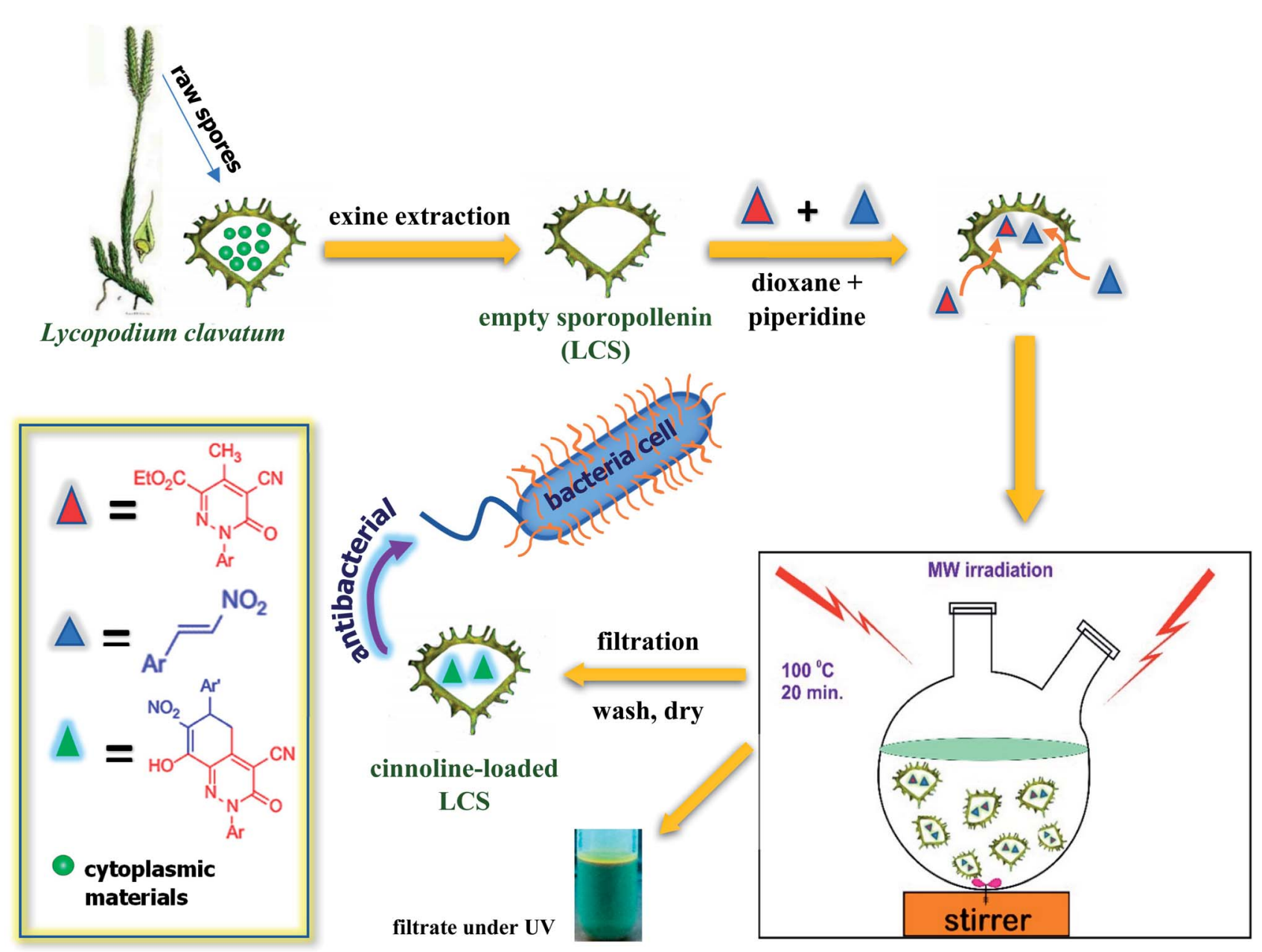

Scheme 1 Schematic presentation of our technique for the in situ green syntheses of a novel cinnoline derivative inside the natural sporopollenin microcapsules via a microwave assisted reaction. 


\section{Experimental}

\subsection{Materials}

Lycopodium clavatum L. (common club moss) spores $(27 \mu \mathrm{m}$ type) were purchased from Fagron, UK. Ethanol, 1,4-dioxane and piperidine were purchased from Sigma-Aldrich (St. Louis, MO, USA). Double sterilised Milli-Q water was used throughout the experiments.

\subsection{Microwave-assisted synthesis of cinnolines inside sporopollenin microcapsules}

Protein-free LCS microcapsules were extracted from the raw Lycopodium clavatum L. spores using a method described elsewhere. ${ }^{18,21}$ Briefly, raw spores were successively treated with acetone, $\mathrm{KOH}$ and ortho-phosphoric acid to get rid of the core cytoplasmic materials and eventually nitrogen-free LCS exines, ready for reactants loading, were obtained. Different materials can be easily loaded into the sporopollenin by passive diffusion, vacuum loading or via using compressed tablets. ${ }^{17,18}$ We have used the passive diffusion protocol in the current study to load the reactant components. The microwave assisted chemical reaction was conducted for the formation of the cinnoline derivative 8-hydroxy-7-nitro-6-(3-nitrophenyl)-3-oxo-2-( $p$-tolyl)2,3,5,6-tetrahydrocinnoline-4-carbonitrile 3 inside the LCS as shown in Scheme 2. The ethyl 5-cyano-4-methyl-6-oxo-1-( $p$-tolyl)1,6-dihydropyridazine-3-carboxylate 1 was prepared according to the previously reported methods..$^{35-37}$ The 1-nitro-2phenylethylene 2 was prepared following standard procedures described elsewhere. ${ }^{38}$ Equimolar $(50 \mathrm{mM})$ of both compound 1 and 2 was prepared in $5 \mathrm{ml}$ dioxane and the reactant mixture was stirred for $10 \mathrm{~min}$ until fully dissolved. The mixture was transferred into a $25 \mathrm{ml}$ two-neck round-bottom flask and a 30 $\mu \mathrm{l}$ of piperidine was then added and stirring was sustained for further $5 \mathrm{~min}$. Then, a $100 \mathrm{mg}$ of dry LCS microcapsules were suspended in the reaction mixture and stirred for $2 \mathrm{~h}$ at $500 \mathrm{rpm}$ to allow passive loading of the reactants into the cavities of the LCS. The mixture was then irradiated by MW in a Milestone Microwave Labstation (Italy) under reflux at $100{ }^{\circ} \mathrm{C}$ for $20 \mathrm{~min}$. The cinnoline (cinn)-loaded LCS microparticles were then collected by filtering through a Whatman filter paper $11 \mu \mathrm{m}$, washed with pure ethanol to remove surface adhered products

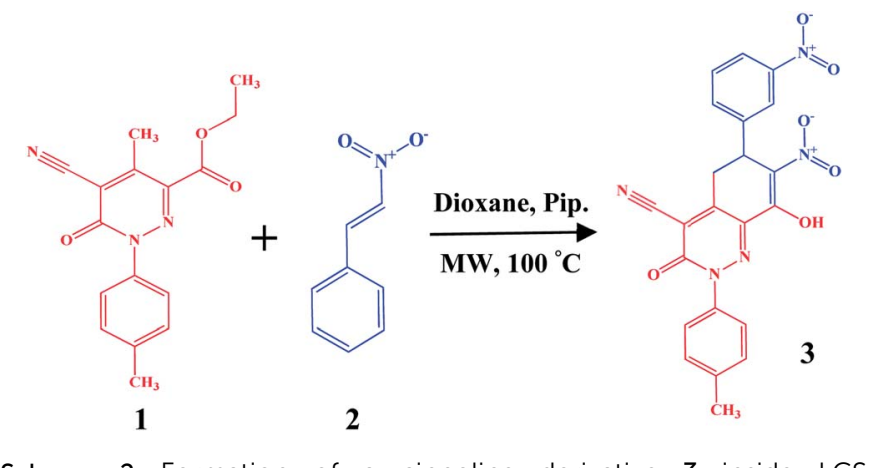

Scheme 2 Formation of a cinnoline derivative 3 inside LCS microcapsules. and finally dried at $75{ }^{\circ} \mathrm{C}$ until constant weight and stored at 2$8{ }^{\circ} \mathrm{C}$ until further use. The filtrate, after ethanol washing, was collected and solvents were removed under reduced pressure and the solid cinnoline 3 product was collected by filtration, recrystallised from ethanol and dried at $75{ }^{\circ} \mathrm{C}$ for further use.

\subsection{Confocal laser scanning microscopy (CLSM) analysis}

CLSM analysis of the empty LCS and cinn-loaded LCS microcapsules was performed using a Carl Zeiss LSM710 (Germany) confocal microscope. Laser excitation lines $405 \mathrm{~nm}, 488 \mathrm{~nm}$ and $543 \mathrm{~nm}$ with a Plan-Abochromat $63 \times / 1.4$ oil DIC (differential interference contrast) M27 objective lens were used. Fluorescence from plain LCS and cinn-loaded LCS were collected with photomultiplier having the following emission filters: 410-481, 491-541 and 595-735. A plane mode scanning with a 6.3 and $12.6 \mu$ s pixel dwell and a beam splitter (MBS 488/543/633, MBSInVis-405) were used. At least three images were captured for each sample. Images were recorded at either a specific slice position or as Z-stack collection through the sample. All images and videos were processed under the same conditions, for comparison, using ZEN 2009 and LSM image browser software (Zeiss, Germany).

\subsection{UV-VIS, FTIR, TGA, SEM, XRD and LCMS analyses}

The maximum absorbance of cinnoline solutions was measured using (Unicam He $\lambda$ ios double beam UV-Vis Spectrometer). The fourier-transform infrared spectroscopy (FTIR) spectra were obtained in the $650-4000 \mathrm{~cm}^{-1}$ range by a Perkin-Elmer 100 spectrometer. Samples were ground with anhydrous potassium bromide (spectrosol grade) to obtain disks to a ratio of $1 / 9(\mathrm{w} / \mathrm{w})$. FTIR spectra were a result of 3 scans against a background. Thermogravimetric analysis (TGA) curves were obtained using TA Instruments (TGA Q50 Thermogravimetric Analyzer) with a heating rate of $10{ }^{\circ} \mathrm{C} \min ^{-1}$ up to $700{ }^{\circ} \mathrm{C}$ under $\mathrm{N}_{2}$ flow. Scanning electron microscope (SEM) analysis was obtained using JSM-5400 LVJEOL (Japan) after coating all samples with $20 \mathrm{~nm}$ gold with gold sputter (JEOL JFC-1100E). Images were taken with an acceleration voltage of $5 \mathrm{kV}$ at various magnifications. The powder X-ray diffraction (XRD) pattern was obtained using PANalytical empyrean diffractometer at $25{ }^{\circ} \mathrm{C}$ employing High score plus software. Agilent 6420 Series Triple Quadrupole LC/MS system was used to measure the mass-tocharge $(\mathrm{m} / \mathrm{z})$ ratios of the ions formed from the released cinnoline compound. The electrospray ionisation mass spectrometry (ESI-MS) in a negative ionisation mode was applied (more details are shown in ESI $\dagger$ ). Optical images were recorded by a Nikon microscope fitted with PHD-5MP digital camera (MicroCam, Egypt) and the images were processed using microvision software.

\subsection{Antibacterial activity}

The antibacterial activity of pure cinnoline derivative 3 and the cinn-loaded LCS was investigated by the standard disc diffusion assay. ${ }^{39}$ Two bacterial strains were used, namely Pseudomonas aeruginosa (Gram-negative), and Bacillus cereus (Gramnegative). The strains were obtained from the Spanish Type 
Culture Collection (CECT; Valencia, Spain). The standard bacterial inoculum suspension $\left(10^{6} \mathrm{cfu} \mathrm{ml}^{-1}\right)$ (colony forming unit) were firstly evaluated by measuring optical density (OD) at $600 \mathrm{~nm}$ and then $100 \mu \mathrm{l}$ of the bacterial inoculum were over streaked on the surface of the Gibcos LB broth/agar medium using sterile cotton swab and grown overnight at $37{ }^{\circ} \mathrm{C}$ on a rotary shaker $(150 \mathrm{rpm})$. A $6 \mathrm{~mm}$ diameter double sterilised paper discs (Model SR-24A-ADVPB, Consolidated, Machine Corporation, Boston, MA, USA) were placed on the prepared Gibcos LB agar plates. A $10 \mu \mathrm{l}$ from pure cinnoline 3 or the cinn-loaded LCS (with MIC conc. value for each) were dropwise added into the filter discs. All discs were completely dried and placed onto the plates and incubated at $37{ }^{\circ} \mathrm{C}$ for $24 \mathrm{~h}$. The antibacterial activity for each pure cinnoline derivative 3 and the cinn-loaded LCS was then evaluated by measuring the zone of inhibition around the filter discs for the tested bacteria. Discs of empty LCS were used alone as controls. All experiments were performed in triplicates.

\section{Results and discussion}

\subsection{Synthesis of cinnoline derivatives inside sporopollenin via microwave irradiation}

We have devoted our efforts to carry out the MW assisted reaction, presented in Scheme 2, for the formation of cinnoline
3 in the interior cavities of the natural LCS capsules. Dioxane and piperidine were found to be the optimum solvent and base, respectively, for the formation of the highest yields of different cinnoline derivatives. ${ }^{34}$ Prior loading the reactants into the LCS, the inner cytoplasmic materials have been extracted from the raw Lycopodium clavatum L. spores to obtain the outer sporopollenin exines, which are free from allergens. The extraction protocol used in the current study was known to produce sporopollenin, from the same pollen species studied here, with $0 \% \mathrm{~N}_{2} \cdot{ }^{15,17,18,21}$ All the components of the reaction were loaded into the empty LCS microcapsules via passive diffusion protocol at room temperature. Fig. 1a and b represents optical images of the LCS immediately after the end of the MW reaction (20 min) where the needle-like crystals of the produced cinnoline 3 can be clearly seen around the loaded LCS capsules, indicative of the successful MW reaction. The excess of the reaction product was removed by filtration and washing with pure ethanol to collect the cinn-loaded LCS capsules as seen in Fig. 1c and d. Solid cinnoline $\mathbf{3}$, green in colour, was recovered from the filtrate after removing the solvents and recrystallisation from ethanol. Hence, no waste was left from the whole process. A movie was recorded focusing on a single cinn-loaded LCS capsule $(\mathrm{V} 1, \mathrm{ESI} \dagger)$ immediately after washed with ethanol where a Brownian motion was realised of sub-micrometre sized particles inside the LCS which is a characteristic for colloids.
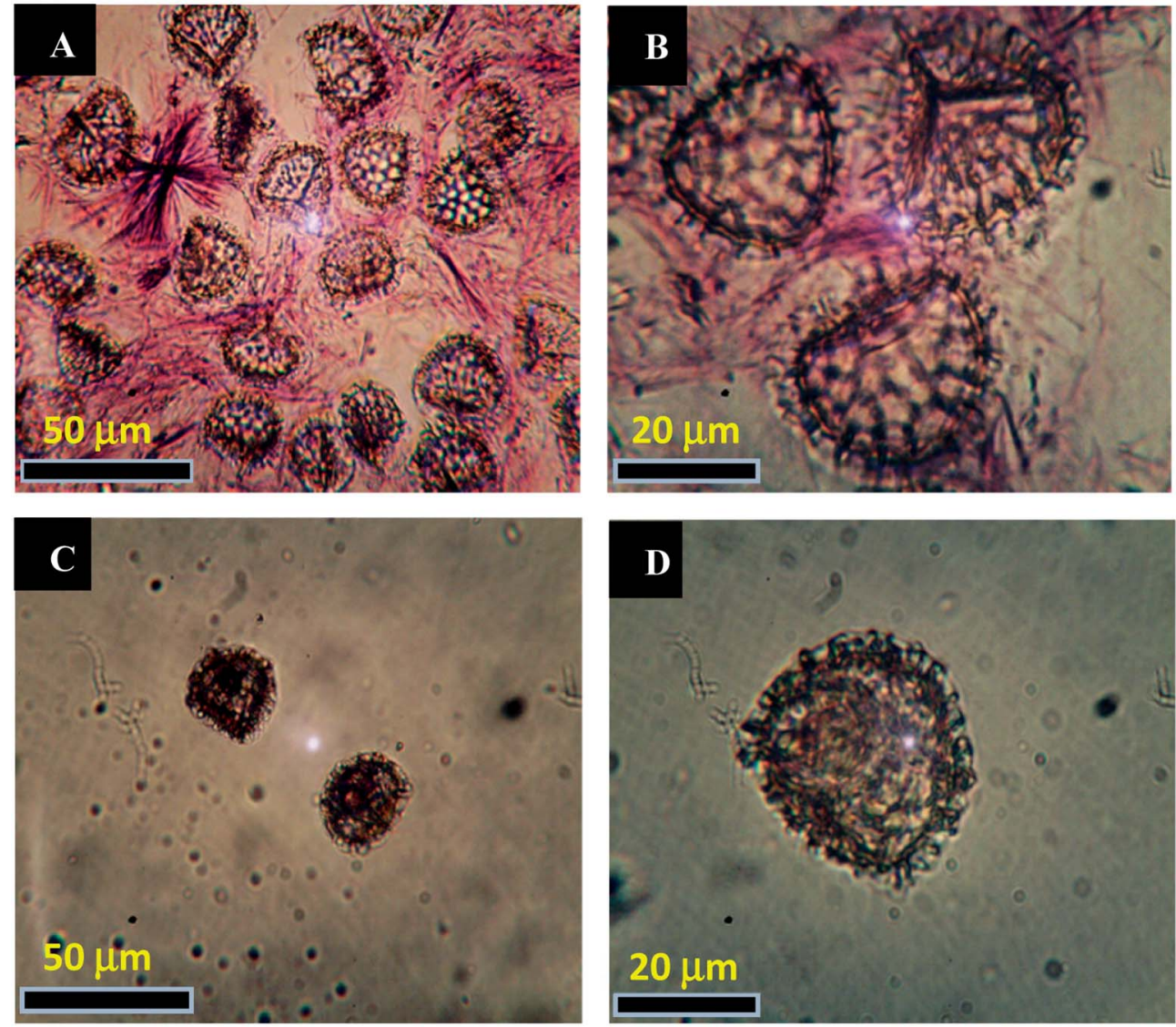

Fig. 1 Optical images of LCS microcapsules. (A and B) Immediately after the end of the MW assisted reaction showing the LCS surrounded by clear crystals of the formed cinnoline 3. (C and D) Cinn-loaded LCS after washing with ethanol. 

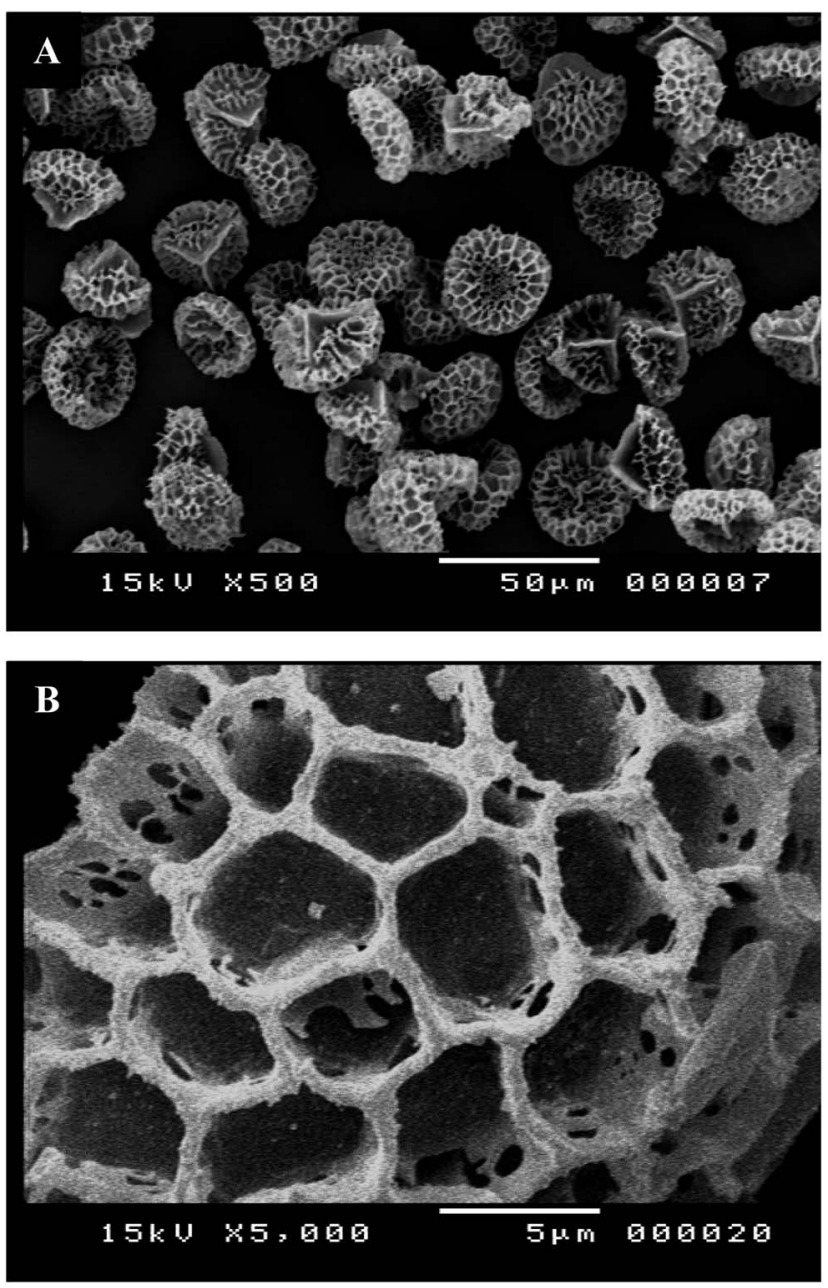

Fig. 2 SEM images of LCS microcapsules showing the surface morphological characterisation. (A) Empty LCS before the reaction and (B) Cinn-loaded LCS after MW assisted reaction at $100^{\circ} \mathrm{C}$ for $20 \mathrm{~min}$

We envisage that these particles might be the trapped cinnoline 3.

It was crucial to verify that the LCS capsules retain their surface structure and there were no reaction products accumulate on their surfaces. Therefore, surface SEM morphology of the LCS was studied before and after the MW synthesis reaction. Fig. 2a represents an SEM image of the extracted empty LCS before the reaction. One can see that the empty LCS is characterised by obvious reticular microstructure and ornamentation, having hexagonal cells resembling the honey comb. The trilete scars (Y shaped) can also be seen which are characteristic for this particular pollen species. It was reported that these LCS can preserve their native microstructure after the extraction process, indicative of the robust resistance of these unique biopolymer to harsh chemical conditions. ${ }^{7,18}$ Fig. $2 \mathrm{~b}$ shows an SEM image focusing on the detailed surface of a cinn-loaded LCS after the MW reaction. It is obvious that the structural integrity of the capsule is evident with no indication of any residual accumulating within their reticular cells. Therefore, the MW reaction condition did not affect the characteristic natural surface microstructure of these LCS.

The available routes for the dissolved reactants to diffuse into the cavities of the LCS are probably through the nanochannels and/or the trilete scars on their surfaces. ${ }^{18,40}$ It was reported that the transfer of the male genetic materials takes place through the apertures of the mature aperturate pollens, whereas for nonaperturate pollens it takes place through the whole exine (sporopollenin) layer. ${ }^{41,42}$ Interestingly, using colloidal iron as a tracer, the exine layer was found to be crossed in several mature spore species by radial microchannels of around $25 \mathrm{~nm}$ in diameter. ${ }^{41-43}$ The mechanism by which different materials transfer in or out the sporopollenin through these apertures and/or the nanochannels is not exactly known since the full chemistry of the sporopollenin is largely unraveled. $^{2}$

Following optical and SEM morphological characterisations, which cannot solely confirm the formation of cinnoline $3 \mathrm{in} \mathrm{situ}$, we have used CLSM analysis to verify its formation. Fig. 3a shows a CLSM image of empty LCS microcapsules before loading any reactants inside. Empty large cavities, ready for loading, can be clearly seen as black cores with no fluorescence, indicative of the effectiveness of the extraction protocol used. However, the exine shells around these empty voids have fluoresced since it is known that natural spores exhibit autofluorescence over a wide excitation wavelength. ${ }^{7,16}$ CLSM images
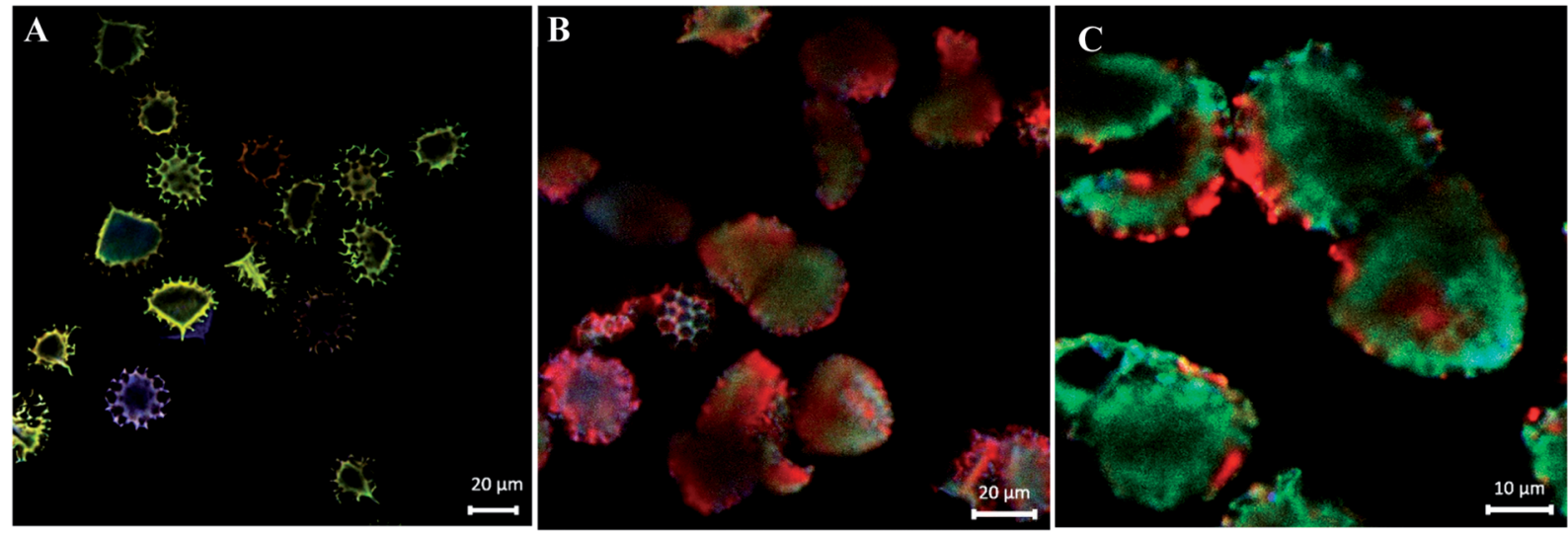

Fig. 3 Overlay CLSM images of (A) empty LCS microcapsules, (B) cinn-loaded LCS microcapsules at a random focus position and (C) cinnloaded LCS at a slice $14.6 \mu \mathrm{m}$ depth, showing the successful formation of cinnoline 3 in situ via a microwave assisted synthesis reaction. 


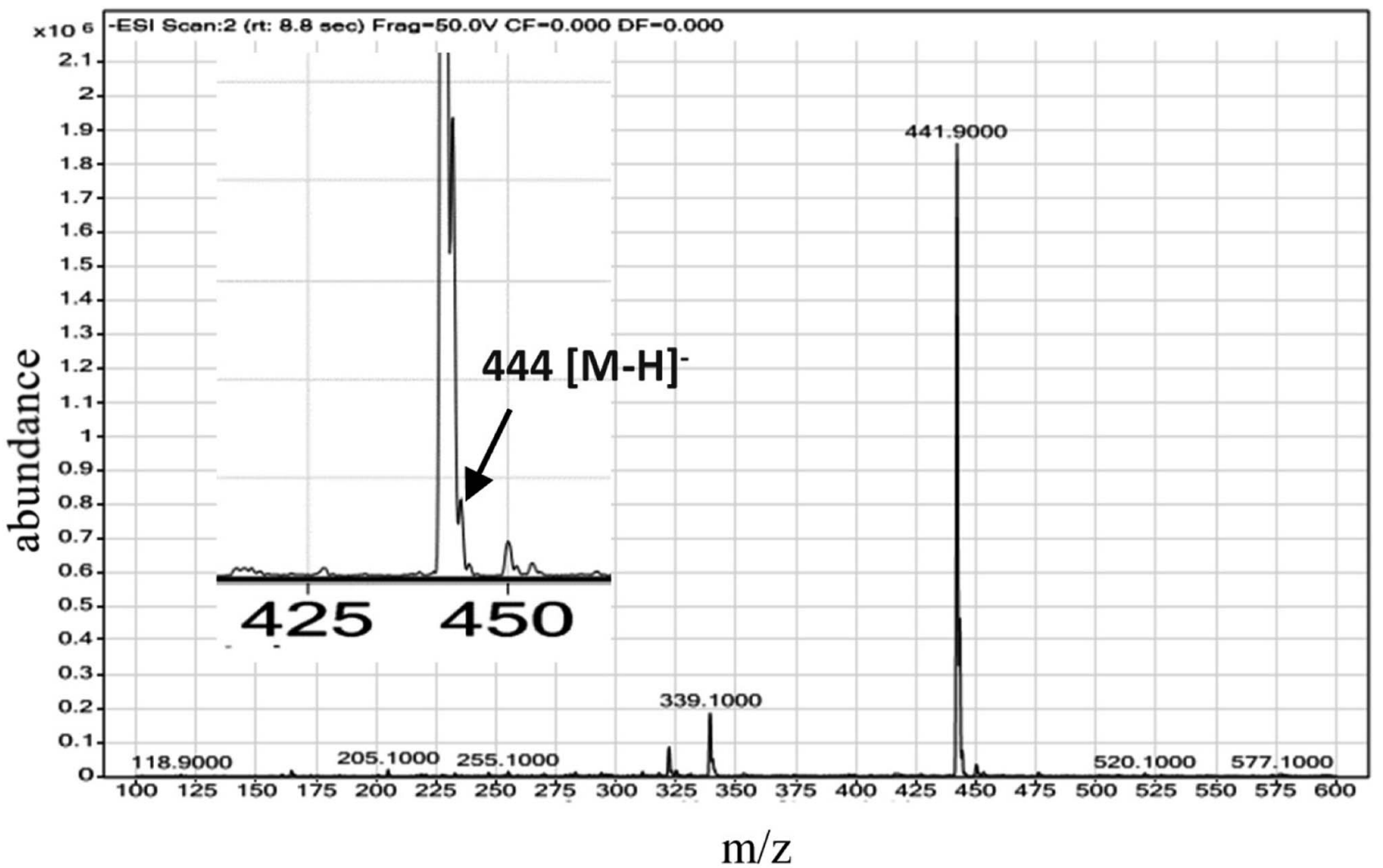

Fig. 4 ESI mass spectrum of cinnoline 3 released from cinn-loaded LCS microcapsules. The inset is detailed peaks within $425-450 \mathrm{~m} / \mathrm{z}$ scale for clarity.

were recorded at blue, green and red excitation channels as well as DIC and overlay images at a middle slice of empty LCS microcapsules, see Fig. S1 (ESI $\dagger$ ). Fortunately, we found that pure cinnoline 3 compound exhibits interesting optical properties (discussed in the next section). Therefore, there was no need to label it since it will fluoresce upon excitation as we expected. Fig. 3b and c show overlay CLSM images of washed and dried cinn-loaded LCS microcapsules after the MW reaction at random and fixed focus position (14.6 $\mu \mathrm{m}$ depth), respectively. It is obvious that strong green-red fluorescence is evident inside the LCS microcapsules with no significant debris shown surrounding them, indicative of successful formation of

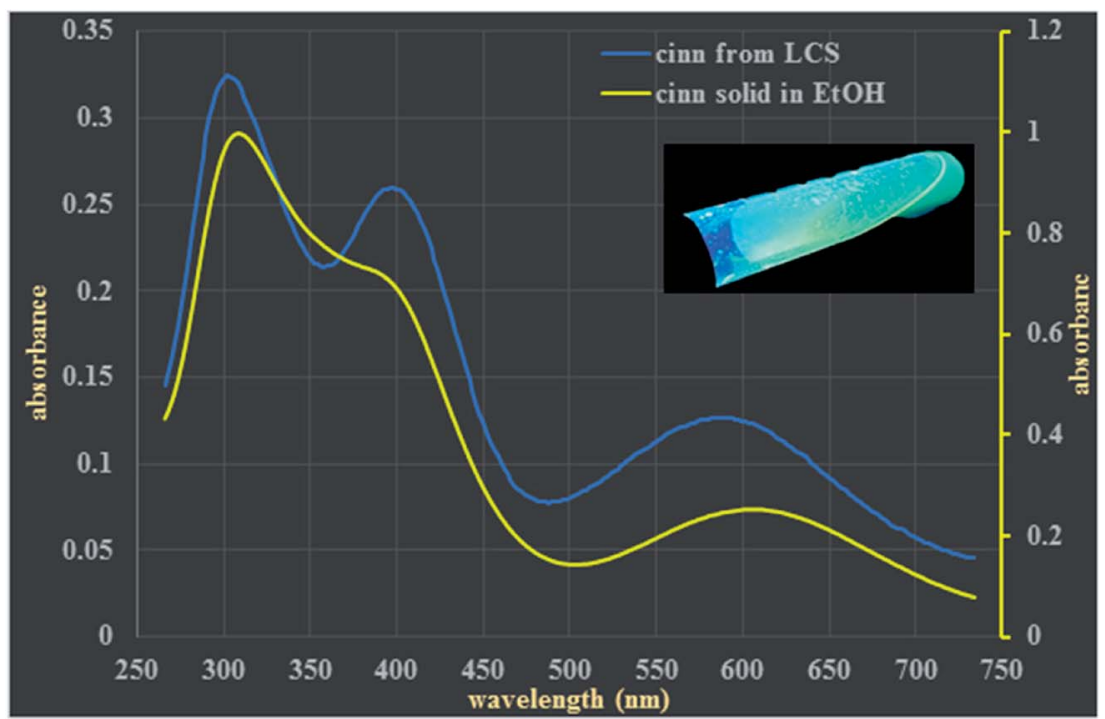

Fig. 5 UV-VIS spectra of cinnoline 3 in pure form and after released from cinn-loaded LCS in ethanol. The inset is a digital image of the filtrate left after the MW reaction under a long wavelength UV lamp. 

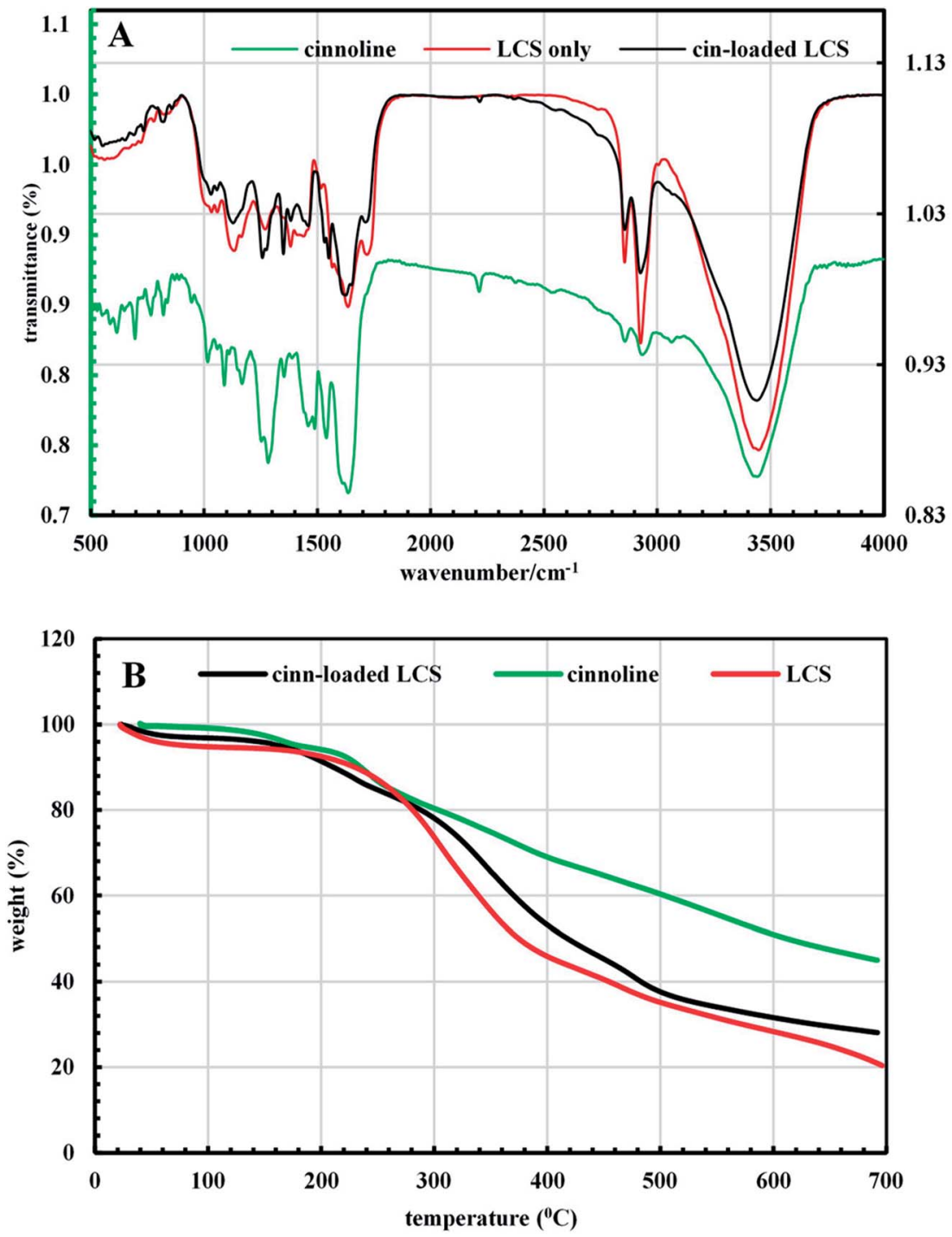

Fig. 6 (A) FTIR spectra and (B) TGA thermogram of pure cinnoline 3, empty LCS and cinn-loaded LCS microcapsules.

cinnoline 3 in situ via the MW assisted reaction shown in Scheme 2. It is worth stressing that the cinn-loaded LCS microcapsules were washed carefully with pure ethanol to remove any residual materials deposited on their surfaces as evident in Fig. 1c and d and 2b. To further support the formation of cinnoline 3 inside the LCS, more CLSM images at different excitation channels are shown in Fig. S2 (ESI $\dagger$ ). In addition, CLSM images recorded in z-stack mode at different depth positions for the cinn-loaded LCS are shown in Fig. S3 along with z-stack videos (V2 and V3 in ESI†). We have also realised that most of the empty LCS microcapsules were filled with the fluorescent cinnoline $\mathbf{3}$ compound. It was reported that phenolic and aliphatic compounds are the two key known components of the natural sporopollenin. The former include trans ferulic acid and $p$-coumaric acid, whereas the latter include saturated and unsaturated fatty acids. ${ }^{44-46}$ The phenolic components in the sporopollenin have been shown to absorb $\mathrm{UV}$ radiation in the $(280-315 \mathrm{~nm})$ and (315-400 $\mathrm{nm})$ range, providing a protection of the cytoplasmic core materials against UV damage. ${ }^{46}$ We envisage that these phenolic components, and probably others, contribute to the autofluorescence of the sporopollenin seen in Fig. $3 \mathrm{a}$.

In this respect, it was interesting to see whether the interior surface of the sporopollenin has any effect on the MW synthesis reaction and its products. Using LCMS analysis, we have tested the integrity of the cinnoline 3 formed within the LCS microcapsules and whether there were any interactions taking place in the inner surface of the sporopollenin as well as within the route used for releasing the trapped materials, for example, the nanochannels. The dried cinn-loaded LCS microcapsules were stirred in pure ethanol for $5 \mathrm{~min}$ to allow the release of cinnoline $\mathbf{3}$, then the first filtrate was discarded. This step was repeated once more with the dried microcapsules left from the first step but with HLPC grade methanol and the second filtrate was tested for the relative mass of cinnoline 3 as seen in the MS Fig. 4 . As revealed from the ESI- 
MS chart, the $[\mathrm{M}-\mathrm{H}]^{-}$peak occurred at $m / z=444$ which represents cinnoline $3\left(\mathrm{C}_{22} \mathrm{H}_{15} \mathrm{~N}_{5} \mathrm{O}_{6}\right.$, calculated mass 445.1). Therefore, the results obtained from LCMS analysis further confirms the formation of cinnoline 3 within the LCS and, more importantly, it was released intact. This in turn can offer another interesting feature of the natural LCS besides their known unique properties. Our findings also support the feasibility of our technique to use these natural LCS as MW microreactors for other important organic or inorganic synthesis inside their cavities. In addition of decent thermal stability of LCS (discussed later), it is also possible to conduct MW reaction at high pressures since it was shown that these LCS can withstand high hydrostatic pressure of $10 \mathrm{GPa} .{ }^{45}$ The retrieval of undamaged materials from microcapsules is vital for potential applications of these LCS microreactors, and probably others, that require the protection of labile materials from denaturation or from harsh environmental conditions. It is important to note that the chemical and physical properties of the nanochannels, as one route for material passage, on sporopollenin showed variations depending on the plant species. Bohne et al. ${ }^{47}$ reported that, for tripartite sporopollenin extracted from Pine pollens, there was a physical barrier to non-electrolytes with a Stokes' radius $>4 \mathrm{~nm}$ resulting from inhomogeneity in the size of the nanochannels. The authors also suggested that there was another electrostatic barrier to electrolytes owing to the specific functional groups inside these nanochannels, hence their charge. In contracts to the findings of Bohne et al. ${ }^{47}$ the sporopollenin extracted from Lycopodium clavatum did not show such barriers as indicated in several studies. ${ }^{7,17,18,21}$

\subsection{Physico-chemical characterisations of pure cinnoline 3 and cinn-loaded LCS microcapsules}

Following the verification of the feasibility of our technique (Scheme 1), it was pertinent to evaluate some physico-chemical properties of both the pure cinnoline 3 derivative and the cinnloaded LCS microcapsules. Combined with their enormous applications, the optical properties of heterocycles, particularly cinnolines, received considerable interests. ${ }^{31,48-50}$ The solid cinnoline 3 collected from the filtrate after the MW synthesis reaction was green in colour and showed fluorescence properties as revealed from CLSM analysis discussed above. Fig. 5 shows UV-vis spectra for cinnoline 3 either dissolved as a pure form in ethanol or released from the cinn-loaded LCS. Concentrated pure cinnoline 3 in ethanol exhibited two $\lambda_{\max }$ absorption peaks at 310 and $610 \mathrm{~nm}$ together with a small shoulder at $400 \mathrm{~nm}$. The cinnoline 3, with a much lower concentration, released from the microcapsules showed three peaks at 304, 400 and $594 \mathrm{~nm}$. The inset digital image in Fig. 5 shows a tube of the filtrate left after the MW reaction under a long wavelength of a UV lamp where the strong green fluorescent is evident. More digital images of these solutions are shown in Fig. S4 (ESI $\dagger$ ). Generally, it was shown that UV-VIS absorption behaviours of heterocycles are very sensitive to solvents and substituents. ${ }^{48}$ Whilst our preliminary results of the optical properties of these novel cinnoline derivatives are promising, more investigations are required which are currently undertaken.

We next performed a comparison of FTIR superimposed spectra of pure cinnoline 3, empty LCS and cinn-loaded LCS (Fig. 6a) to explore whether there are any interactions between the components and any changes resulted from the MW assisted reaction. As revealed from Fig. 6a, empty LCS exhibited OHstretching peak at $3447 \mathrm{~cm}^{-1}, \mathrm{CH} n$ stretching peaks at 2926 and $2855 \mathrm{~cm}^{-1}, \mathrm{C}=\mathrm{O}$ group at $1719 \mathrm{~cm}^{-1}, \mathrm{C}-\mathrm{O}$ stretching peaks at 1133 and $1269 \mathrm{~cm}^{-1}$. Pure cinnoline 3 showed the following characteristic peaks: aromatic $\mathrm{C}-\mathrm{H}$ stretching peak at $3062 \mathrm{~cm}^{-1}, \mathrm{C}=\mathrm{C}$ stretching at $1635 \mathrm{~cm}^{-1}$, aromatic $\mathrm{C}-\mathrm{H}$ inplane bending peaks at $116-1253 \mathrm{~cm}^{-1}, \mathrm{C}-\mathrm{H}$ out of plan (oop) peaks at $695-890 \mathrm{~cm}^{-1}, \mathrm{~N}-\mathrm{O}$ stretching peaks at 1539 and

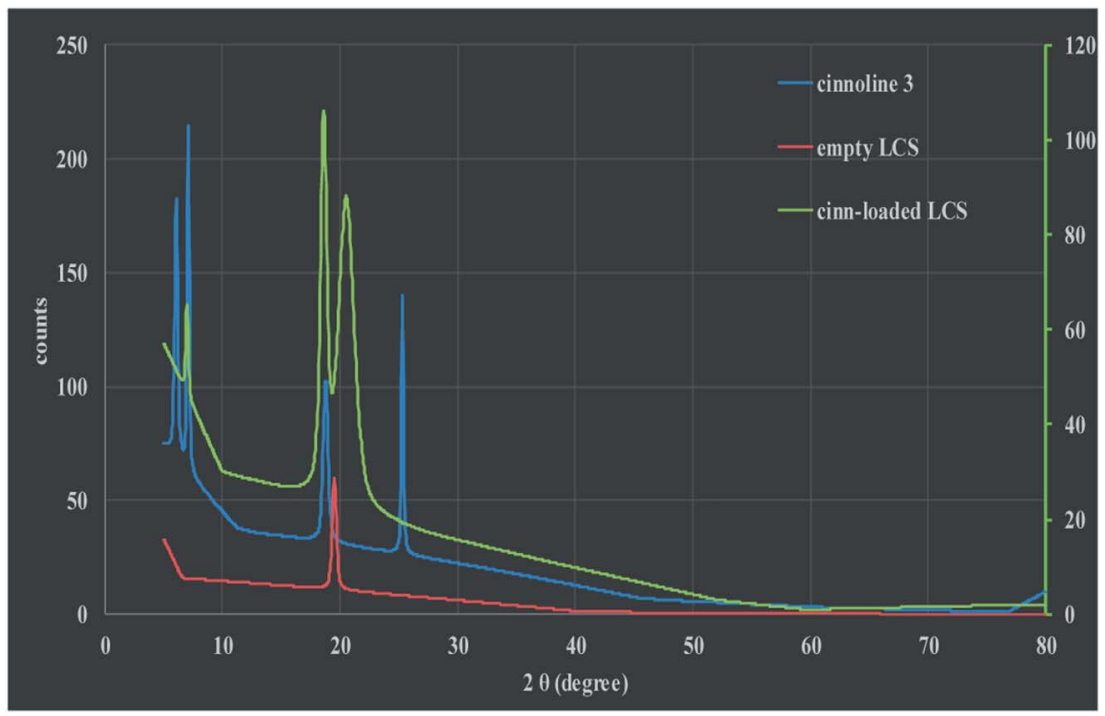

Fig. 7 Calculated intensity (I calc $)$ profile of XRD of cinnoline 3, empty LCS and cinn-loaded LCS at $25^{\circ} \mathrm{C}$. 
$1353 \mathrm{~cm}^{-1}$. It can be seen from FTIR spectrum of cinn-loaded LCS in Fig. 6a that there was no significant change in its spectrum when compared to that of the empty LCS apart from the appearance of a peak at $1350 \mathrm{~cm}^{-1}$ and the peak at 1550 became sharper. This might indicate that cinnoline is mostly encapsulated within the LCS and not on their surfaces. Similar observations were reported for ascorbic acid ${ }^{51}$ and ibuprofen ${ }^{19}$ encapsulated into similar sporopollenin. In another study, it was reported that pollen grains exhibit a strong light scattering, owing to their size and spatial orientation, resulted in anomalous FTIR spectra which can significantly interfere with and alter the signals of chemical absorption. ${ }^{52}$ In this respect, our results suggested that the characteristic surface functional groups of the LCS have not significantly altered during and after the MW reaction.

TGA data gives useful information about the decomposition behaviour of materials for their potential applications. Fig. $6 \mathrm{~b}$ shows the TGA graph from $20-700{ }^{\circ} \mathrm{C}$ for the materials studied here. Generally, the release of the physically adsorbed water occurs in the first decomposition step at around $100{ }^{\circ} \mathrm{C}$. The empty LCS thermally decomposed in three steps. Around $49 \%$ of the weight loss of empty LCS occurred at $400{ }^{\circ} \mathrm{C}$ and the temperature at which the weight loss begins (onset temperature) was $250{ }^{\circ} \mathrm{C}$. The pure cinnoline 3 exhibited three steps decomposition and its onset temperature was $156.3{ }^{\circ} \mathrm{C}$. Cinnoline 3 showed good stability at higher temperature where the weight loss was $55 \%$ at around $700{ }^{\circ} \mathrm{C}$, whereas it was $80 \%$ for empty LCS. Cinn-loaded LCS decomposed in four steps and the onset temperature was $159.4{ }^{\circ} \mathrm{C}$, indicative of the incorporation of cinnoline 3 inside the LCS microcapsules. The difference in the final weight loss between the empty and cinn-loaded LCS suggested the loading of cinnoline 3 . In addition, the loading content can be estimated from that difference from TGA data as reported by Kotcherlakota et al. ${ }^{53}$ From Fig. 6b the estimated cinnoline 3 loading was around $10 \%$. Also, it can be noticed that the presence of cinnoline 3 enhanced the thermal stability of

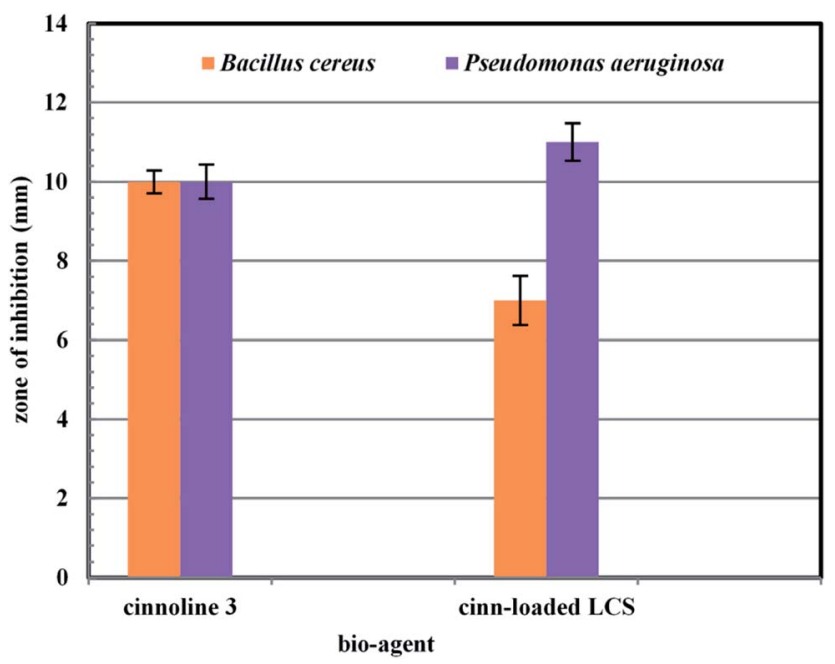

Fig. 8 Antibacterial activity of cinnoline 3 and cinn-loaded LCS microcapsules. the cinn-loaded LCS. Interestingly, we have carried out TGA analysis for cinn-loaded LCS just after drying at room temperature and found that LCS are able to accommodate solvent of around $76 \%$ of its initial mass as reflected from the TGA Fig. S5 (ESI $\dagger$ ). That is why we have dried the loaded microcapsules after the MW reaction at $75^{\circ} \mathrm{C}$ to get rid of any encapsulated solvents even if they seem dry.

We have also performed XRD analysis of the materials used in this study and the data are shown in Fig. 7 and S6 (ESI $\dagger$ ). Fig. 7 represents the calculated intensity $\left(I_{\text {calc }}\right)$ profile for XRD pattern of pure cinnoline 3, empty LCS and cinn-loaded LCS. The empty LCS microcapsules showed a typical amorphous structure with a characteristic peak at $2 \theta=19.48^{\circ} .^{54}$ The observed intensity XRD profile for empty LCS is shown in Fig. S6 (ESI $\dagger$ ) where the broad peak is obvious. Cinnoline 3 exhibited some crystalline feature where four peaks can be seen. Nevertheless, cinnoline 3 still has amorphous structure with lower peak noises compared to empty LCS (Fig. S6, ESI $\dagger$ ). Cinn-loaded LCS showed amorphous structure with three peaks, two of them are characteristic of cinnoline 3 (at $2 \theta=7.07$ and $18.75^{\circ}$ ) as evident in Fig. 7, indicative of the existence of cinnoline 3 in the loaded cinn-loaded. The results obtained from XRD analysis further confirm the feasibility of our technique.

\subsection{Antibacterial activity}

To the best of our knowledge, there were no previous reports examined the antibacterial activity of cinnoline 3 or cinn-loaded LCS microcapsules. We present a preliminarily study of the antibacterial activity of both cinn-loaded LCS and the pure cinnoline 3 as shown in Fig. 8 and S7 (ESI $\dagger$ ). A concentration of $10 \mu \mathrm{g} \mathrm{ml}^{-1}$ of each was used. The antibacterial results showed that no antibacterial activity was noticed for empty LCS microcapsules. Intriguingly, a promising antibacterial activity of both cinnoline 3 and cinn-loaded LCS against both Gram positive and Gram negative bacteria were observed. In this respect, we envisage that the unique natural structural morphology of LCS surfaces might help the adherence with the bacterial cell wall leading to an increase in the time of contact, thus enhancing the antibacterial activity. However, this hypothesis requires more investigations which are currently underway. This is somewhat similar to the de novo contacts of pollen grains to the female stigma cells which was reported to be governed possibly by specific interactions involved the lipophilic molecules on the pollen exine. ${ }^{55}$

\section{Conclusions}

Bridging the chasm between microencapsulation strategies and the use of greener chemical reactions, we have achieved a green and efficient protocol for MW assisted synthesis of novel cinnoline derivatives inside the cavities of natural sporopollenin microcapsules extracted from Lycopodium clavatum spores for the first time. Given their highly consistency in sizes, resilience to harsh environmental conditions and unique morphology for specific plant species, sporopollenin can act as robust MW microreactors. To conduct the MW reaction, the reactants were 
simply loaded into the empty LCS via a passive diffusion technique and the reaction was promoted by controlled MW irradiation. Several techniques were used to confirm the formation of cinnoline derivative inside the LCS and to characterise both the pure product and the cinn-loaded LCS microcapsules. The surface of the cinn-loaded LCS did not show any significant accumulation of residual materials, indicative of the efficiency of the technique as revealed from SEM and FTIR analysis. The CLSM analysis further confirms the formation of cinnoline derivative inside the LCS. Crucial for potential applications, we showed that cinnoline derivative was recovered from the cinnloaded LCS without any alternation as revealed from the LCMS analysis. The pure cinnoline derivative exhibited promising optical properties with decent fluorescent which could be extended for use in several potential applications such as optoelectronics and organic luminescence materials. We demonstrated that the pure cinnoline and cinn-loaded LCS show promising antibacterial activity against Gram-positive and Gram-negative bacterial strains. We present in this study a proof of concept, however our technique can be applied using other spore and sporopollenin species to efficiently synthesise different organic or inorganic materials via controlled MW irradiation reactions. In addition, our protocol can pave the way for several potential applications such as materials and pharmaceutical sciences in a more benign route.

\section{Conflicts of interest}

There are no conflicts of interest to declare.

\section{Notes and references}

1 T. Ariizumi and K. Toriyama, Annu. Rev. Plant Biol., 2011, 62, 437-460.

2 G. Mackenzie, A. N. Boa, A. Diego-Taboada, S. L. Atkin and T. Sathyapalan, Front. Mater., 2015, 2, 66.

3 F. Ahlers, H. Bubert, S. Steuernagel, R. Wiermann and Z. Naturforsch, $C, 2000,55,129-136$.

4 P. E. Jardine, W. T. Fraser, B. H. Lomax and W. D. Gosling, J. Micropalaeontol., 2015, 34, 139-149.

5 R. C. Mundargi, M. G. Potroz, J. H. Park, J. Seo, E. L. Tan, J. H. Lee and N. J. Cho, Sci. Rep., 2016, 6, 19960.

6 E. Domínguez, J. A. Mercado, M. A. Quesada and A. Heredia, Grana, 1998, 37, 93-96.

7 A. Diego-Taboada, S. T. Beckett, S. L. Atkin and G. Mackenzie, Pharmaceutics, 2014, 6, 80-96.

8 G. Mackenzie and G. Shaw, Int. J. Pept. Protein Res., 1980, 15, 298-300.

9 S. Barrier, A. Löbbert, A. J. Boasman, A. N. Boa, M. Lorch, S. L. Atkin and G. Mackenzie, Green Chem., 2010, 12, 234240.

10 N. Unlu and M. Ersoz, Sep. Purif. Technol., 2007, 52, 461-469. 11 B. P. Binks, J. H. Clint, G. Mackenzie, C. Simcock and C. P. Whitby, Langmuir, 2005, 21, 8161-8167.

12 S. P. de Souza, J. Bassut, H. V. Marquez, I. I. Junior, L. S. M. Miranda, Y. Huang, G. Mackenzie, A. N. Boa and R. O. M. A. de Souza, Catal. Sci. Technol., 2015, 5, 3130-3136.
13 K. Yusuf, A. Aqel, A. K. F. Dyab, Z. A. Alothman and A. Y. Badjah-Hadj-Ahmed, J. Liq. Chromatogr. Relat. Technol., 2016, 39, 752-761.

14 A. K. F. Dyab, Micro-structured material and method for the preparation thereof, European pat., EP2769995 (B1), 2016.

15 V. N. Paunov, G. Mackenzie and S. D. Stoyanov, J. Mater. Chem., 2007, 17, 609.

16 S. Barrier, A. S. Rigby, A. Diego-Taboada, M. J. Thomasson, G. Mackenzie and S. L. Atkin, LWT-Food Sci. Technol., 2010, 43, 73-76.

17 S. Barrier, A. Diego-Taboada, M. J. Thomasson, L. Madden, J. C. Pointon, J. D. Wadhawan, S. T. Beckett, S. L. Atkin and G. Mackenzie, J. Mater. Chem., 2011, 21, 975-981.

18 S. A. Hamad, A. K. F. Dyab, S. D. Stoyanov and V. N. Paunov, J. Mater. Chem., 2011, 21, 18018-18023.

19 A. Diego-Taboada, L. Maillet, J. H. Banoub, M. Lorch, A. S. Rigby, A. N. Boa, S. L. Atkin and G. Mackenzie, J. Mater. Chem. B, 2013, 1, 707-713.

20 A. Diego-Taboada, P. Cousson, E. Raynaud, Y. Huang, M. Lorch, B. P. Binks, Y. Queneau, A. N. Boa, S. L. Atkin, S. T. Beckett and G. Mackenzie, J. Mater. Chem., 2012, 22, 9767.

21 A. K. F. Dyab, E. M. Abdallah, S. A. Ahmed and M. M. Rabee, J. Encapsulation Adsorpt. Sci., 2016, 06, 109-131.

22 S. A. Hamad, A. K. Dyab, S. D. Stoyanov and V. N. Paunov, MRS Online Proc. Libr., 2013, 1499.

23 M. B. Gawande, S. N. Shelke, R. Zboril and R. S. Varma, Acc. Chem. Res., 2014, 47, 1338-1348.

24 N. Sharma, U. K. Sharma and E. V. Van der Eycken, in Green Techniques for Organic Synthesis and Medicinal Chemistry, ed. W. Zhang and B. W. Cue, John Wiley \& Sons, Ltd, 2018, ch. 17, pp. 441-468, DOI: 10.1002/9781119288152.ch17.

25 C. Cabrele and O. Reiser, J. Org. Chem., 2016, 81, 1010910125.

26 B. Stefańska, M. Arciemiuk, M. M. Bontemps-Gracz, M. Dzieduszycka, A. Kupiec, S. Martelli and E. Borowski, Bioorg. Med. Chem., 2003, 11, 561-572.

27 B. Parrino, A. Carbone, M. Muscarella, V. Spanò, A. Montalbano, P. Barraja, A. Salvador, D. Vedaldi, G. Cirrincione and P. Diana, J. Med. Chem., 2014, 57, 94959511.

28 P. Mishra, A. Middha, V. Saxena and A. Saxena, J. Pharma Biosci., 2016, 4, 64-68.

29 P. Barraja, P. Diana, A. Lauria, A. Passannanti, A. M. Almerico, C. Minnei, S. Longu, D. Congiu, C. Musiu and P. La Colla, Bioorg. Med. Chem., 1999, 7, 1591-1596.

30 T. Mitsumori, M. Bendikov, J. Sedó and F. Wudl, Chem. Mater., 2003, 15, 3759-3768.

31 S. Mayakrishnan, Y. Arun, C. Balachandran, N. Emi, D. Muralidharan and P. T. Perumal, Org. Biomol. Chem., 2016, 14, 1958-1968.

32 T.-Z. Yang, H. Iino and J.-i. Hanna, Liq. Cryst., 2016, 44, 666673.

33 M. J. Sienkowska, J. M. Farrar, F. Zhang, S. Kusuma, P. A. Heiney and P. Kaszynski, J. Mater. Chem., 2007, 17, 1399. 
34 A. A. Hameed, E. K. Ahmed, A. A. A. Fattah, C. K. Z. Andrade and K. U. Sadek, Res. Chem. Intermed., 2017, 43, 5523-5533.

35 M. H. Elnagdi, N. S. Ibrahim, K. U. Sadek and M. H. Mohamed, Eur. J. Org. Chem., 1988, 1005-1006.

36 N. S. Ibrahim, F. M. Abdel-Galil, R. M. Abdel-Motaleb and M. H. Elnagdi, Bull. Chem. Soc. Jpn., 1987, 60, 4486-4488.

37 S. Al-Mousawi, A.-Z. Elassar and M. A. El-Apasery, Phosphorus, Sulfur Silicon Relat. Elem., 2006, 181, 1755-1771.

38 G. Greber, J. Polym. Sci., Part A: Polym. Chem., 1991, 29, 1223.

39 A. Bauer, W. Kirby, J. C. Sherris and M. Turck, Am. J. Clin. Pathol., 1966, 45, 493.

40 R. Wiermann, F. Ahlers and I. Schmitz-Thom, in Biopolymers-lignin, humic substances and coal, ed. M. Hofrichter and A. Steinbüchel, Wiley-VCH, Weinheim, Germany, vol. 1, 2001, pp. 209-227, DOI: 10.1002/ 3527600035.bpol1007.

41 J. Pettitt, Protoplasma, 1976, 88, 117-131.

42 B. M. Gullvåg, Grana, 1966, 6, 435-475.

43 J. R. Rowley, J. J. Skvarla and G. El-Ghazaly, Can. J. Bot., 2003, 81, 1070-1082.

44 P. E. Jardine, F. A. J. Abernethy, B. H. Lomax, W. D. Gosling and W. T. Fraser, Rev. Palaeobot. Palynol., 2017, 238, 1-6.

45 W. Montgomery, C. Potiszil, J. S. Watson and M. A. Sephton, Macromol. Chem. Phys., 2016, 217, 2494-2500.
46 W. T. Fraser, B. H. Lomax, P. E. Jardine, W. D. Gosling and M. A. Sephton, Front. Ecol. Evol., 2014, 2, 12.

47 G. Bohne, E. Richter, H. Woehlecke and R. Ehwald, Ann. Bot., 2003, 92, 289-297.

48 M. Vasilescu, R. Bandula, F. Dumitrascu and H. Lemmetyinen, J. Fluoresc., 2006, 16, 631-639.

49 T. Mitsumori, M. Bendikov, O. Dautel, F. Wudl, T. Shioya, H. Sato and Y. Sato, J. Am. Chem. Soc., 2004, 126, 1679316803.

50 J.-C. Chen, H.-C. Wu, C.-J. Chiang, T. Chen and L. Xing, J. Mater. Chem. C, 2014, 2, 4835-4846.

51 L. J. Blackwell, Sporopollenin exines as a novel drug delivery system, PhD thesis, University of Hull, 2007.

52 B. Zimmermann, M. Bagcioglu, C. Sandt and A. Kohler, Planta, 2015, 242, 1237-1250.

53 R. Kotcherlakota, A. K. Barui, S. Prashar, M. Fajardo, D. Briones, A. Rodriguez-Dieguez, C. R. Patra and S. Gomez-Ruiz, Biomater. Sci., 2016, 4, 448-459.

54 M. Sahin, I. H. Gubbuk and N. Kocak, J. Inorg. Organomet. Polym. Mater., 2012, 22, 1279-1286.

55 G. M. Zinkl, B. I. Zwiebel, D. G. Grier and D. Preuss, Development, 1999, 126, 5431-5440. 